\title{
The Journal of the American Medical Association
}

\author{
Published Under the Auspices of the Board of Trustees
}

\section{THE PROBLEM OF THE CHRONIC CRIPILE* \\ RUSSELL A. HBBS, M.D. NEW YORK}

The increasing interest throughout the country in the problem of the chronic cripple creates an opportunity for this section to be of great service in shaping the future development of work for this class of sufferers. As knowledge of the fact that there are large numbers of people in every community who need orthopedic care increases, and as methods of treatment are perfected, a large number of men are going to select orthopedic surgery as a specialty, and will seek opportunities to equip themselves for this work. It seems to me that we of this section should be able to furnish some suggestions to such nren in determining the nature of their equipment, and the best means of applying it to the needs of the cripple. In answering such questions, the fact must be emphasized that almost all our work in orthopedic surgery has to do with the treatment of the chronic sufferer, which means that our results are long delayed, often requiring years of painstaking care to detail for their acconplishment, and may be secured only by men giving the same enthusiastic consideration to the treatment of these patients which is so easily given to the treatment of the acutely ill. In other words, we have got in some way to keep ourselves working under the inspiration of a chronic enthusiasm as the only means by which we can meet the tremendous difficulties in the treatment of chronic diseases. Not only is such enthusiasm important on the part of the surgeon, but also it is of immense importance in its influence on our patients. If it is tiresome and discouraging to the surgeon to find results long delayed, how much more so is it apt to be to the patients themselves.

Such enthusiasm is of slow growth, and suggests that the orthopedic surgeon as compared with the general surgeon should have a longer period of preliminary preparation. Not only is this necessary for the development of a spirit of enthusiasm but also is it best for technical equipment.

As has been indicated, the results of our methods of treatment are slow in showing, and a thorough knowledge of them cannot be obtained except by studying the individual case for a considerable time in varying conditions of treatment, in the hospital, dispensary and home. The great volume of work for the cripple is

* Chairman's address, read before the Section on Orthopedic Surgery at the Sixty-Seventh Annual Session of the American Medical Associa. tion, Detroit, June, 1916. done in the outpatient or dispensary department, and careful training in this work is as necessary as in the hospital. A large number never have any hospital treatment at all, and those who are admitted to the hospital stay for a short time in comparison with the duration of treatment, and under modern conditions, except in the case of country hospitals for joint tuberculosis, most orthopedic patients are admitted to the hospital for some form of operative treatment. During the past year I have made inquiries of seven clinics in various parts of the country as to the total number of patients treated in each clinic and what proportion of that number were admitted to the hospital for operation. The results of these inquiries have been most surprising, because in the larger clinics, and those which have been longer established, the percentage of patients admitted to the hospital for operation of the total number treated in the dispensary varied from 3 to 10 per cent. In a few instances, the percentage was as high as 18. The average percentage for the whole number was 10 .

These facts have an important bearing on the question we are discussing. If the man's training is only in the hospital, his preparation for the treatment of the 90 per cent. of the cases which never enter the hospital is poor. There is a great deal more written about the 10 per cent. who have operations than the 90 per cent. who do not, and I think therein lies a danger to the future development of our specialty, that in the consideration given to the operative sicle of the work, we may neglect the nonoperative. I am not attempting to raise any question as to the value of operations in orthopedic surgery. There can no longer be any question that the operative aspect of our work is of great importance. All I am concerned about is that the vastly larger volume, the nonoperative, be not neglected. As a matter of fact, the greatest possible operative skill will be defeated in the accomplishment of the greatest amount of good for our patients, unless the man who exercises it has taken pains long before to perfect his equipment in the much more difficult and much more tedious nonoperative work.

Assuming, therefore, the proper training of the man, our second question is, What is the best means of applying it to the needs of the cripple?

This brings us to the question of organization, and I feel sure that from the standpoint of the cripple and his needs, the orthopedic surgeon can be more effective in meeting them in institutions wholly devoted to the treatment of the cripple. There are many communities throughout the country where such work is new and unfamiliar to the profession and the laity, and such places offer wonderful opportunities for men to initiate organizations ideally fitted to the need of the cripple, 
unhampered by the difficulties of attempting to do work for the cripple in a small and insignificant orthopedic department in a general hospital, the spirit and atmosphere of which I do not believe is most helpful to work of this kind.

130 East Thirty-Sixth Street.

\section{TREATMENT OF CONSTIPATION}

BY CONSERVATIVE SURGICAL CORRECTION OF RETARDATIVE DISPLACEMENTS OF THE COLON *

\section{CHARLES A. L. REED, M.D. CINCINNATI}

It is no longer necessary even to affirm that constipation due to displacements, distortions or malformations of the intestines is frequently the cause of various forms of persistent ill health. The demonstration, clinical and pathologic, has been made so frequently and so conclusively that the relationship has become accepted as axiomatic and fundamental. It is no longer a matter of surprise when a progressive practitioner states that constipation is the underlying cause of successively systemic infection, toxemia, acidosis

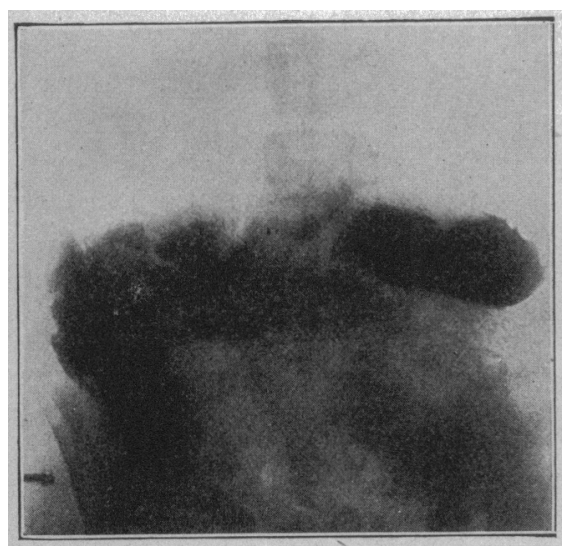

Fig. 1.-Residual barium in stomach after twenty-four hours, visible duodenum, ileac stasis, coloptosis.

and terminal edemas. This new point of view has come to be accepted by the profession as explanatory of many chronic headaches, neuralgias and rheumatisms; of many anemias and neurasthenias; of many digestive troubles, including ulcer of the stomach; of various disorders of the secretory organs, such as Bright's disease and cirrhosis - conditions heretofore recognized as separate diseases but now known to be correlated results of a common origin, or, in other words, the effects of toxic or infectious states often originating in constipation. It is also now recognized that constipation has a contributing causal relation not only to these conditions, but to any or all so-called diseases, the inception or progress of which may be deleteriously influenced by general infection, or by toxemia with resulting acidosis. It is also recognized, thanks to the pioneer work of Bouchard, Glenard and Lane, that logical treatment of these conditions must begin with the correction of the initial anatomic disturbance.

\section{ANATOMIC CAUSES OF CONSTIPATION}

As I have stated in previous publications, I have found, chief among causes of constipation, ptosis with

* Read before the Section on Obstetrics, Gynecology and Abdominal Surgery at the Sixty-Seventh Annual Session of the American Medical Association, Detroit, June, 1916. retardative angulations of the colon, large, flabby and mobile cecum, dilated ascending colon, atrophied transverse colon, redundant transverse colon, retardative angulation at the splenic flexure, retardative angulation with or without adhesion at or near the terminal ileum, and redundancy with or without adhesions of the sigmoid. Each of these conditions, whether coexisting with other of the conditions enumerated or not, is organic; each is associated with tissue or structural change. Each condition must, therefore, find ultimate correction by physical means.

\section{TREATMENT OF MECHANICAL STASIS}

Hygienic and Medicinal.-There may be mechanical distortions of the alimentary canal which, because of their limited extent or because of the nonobstructive character of the resulting angulations, do not cause serious disturbance of function. These are the cases which, because of the limited extent and slight character of the distortion, produce less severe consequences - consequences that may be susceptible of temporary relief by hygienic and medicinal means. These, however, are not the cases that are referred to the surgeon, at least until after the temporizing means shall have failed to arrest the progress of the invalidism. They are not, therefore, the cases that are here under consideration. The cases that are here considered are those in which there is a demonstrated physical impediment to the fecal current, as shown by Roentgen-ray transit studies, and - in which there is a demonstrated relation between the intestinal condition and the constitutional state and, finally, in which previous hygienic and medical treatment has failed either to bring relief or to arrest the progress of the invalidism.

I make this last remark largely in deference to the mistaken notion that it is always better to try everything else before trying surgery,
and I say it in recognition of the fact that the day will come when a condition that is obviously surgical from the start will be given the benefit of surgical treatment before the health has become profoundly and dangerously impaired by dalliance with a futile therapy.

Surgical.-The central idea in surgical treatment of mechanical stasis is, as far as possible, to restore or reestablish the physiologic drainage of the intestines. It may not always be possible to accomplish this purpose for the reason that established pathologic conditions may be of such character as to make impossible the restoration of even a functional equivalent of the status quo ante. A cecum that has become dilated, flabby and hypermobile by distention due to retardative angulation at the hepatic flexure, a transverse colon that has become atrophied by prolonged enervation due chiefly to purgation, a mucous membrane of either the large or small bowel that has become the seat of deep interstitial and follicular infection, as in some cases of epilepsy, and a marked redundancy of any portion of either the colon or the sigmoid are examples of pathologic change that defy functional restoration. 